\title{
Water adsorption on amorphous silica surfaces: A Car-Parrinello simulation study
}

\author{
Claus Mischler ${ }^{1)}$, Jürgen Horbach ${ }^{1)}$, Walter $\mathrm{Kob}^{2)}$, and Kurt Binder ${ }^{1)}$ \\ 1) Institut für Physik, Johannes Gutenberg-Universität Mainz, \\ Staudinger Weg 7, D-55099 Mainz, Germany \\ 2) Laboratoire des Collö̈des, Verres et Nanomatériaux, Université Montpellier II, \\ Place E. Bataillon cc 069, F-34095 Montpellier, France
}

\begin{abstract}
A combination of classical molecular dynamics (MD) and ab initio Car-Parrinello molecular dynamics (CPMD) simulations is used to investigate the adsorption of water on a free amorphous silica surface. From the classical $\mathrm{MD} \mathrm{SiO}_{2}$ configurations with a free surface are generated which are then used as starting configurations for the CPMD. We study the reaction of a water molecule with a two-membered ring at the temperature $T=300 \mathrm{~K}$. We show that the result of this reaction is the formation of two silanol groups on the surface. The activation energy of the reaction is estimated and it is shown that the reaction is exothermic.
\end{abstract}

Keywords: Car-Parrinello molecular dynamics; amorpous silica; surfaces 


\section{INTRODUCTION}

The interaction of water with surfaces of amorphous silica is of great technological interest 1, 2], and thus numerous studies have been devoted to this issue ranging from IR spectroscopy 3, 4, 5, 6, 7, 8] to numerical methods using both ab initio techniques [9, 10, 11, 12, 13, 14, 15] and classical MD simulations [16, 17, 18, 19]. In these studies, the possible existence of small-membered $\mathrm{Si}-\mathrm{O}$ rings on $\mathrm{SiO}_{2}$ surfaces has been intensely discussed since such rings are expected to be the reactive centres for the interaction with water and other molecules. It is well-known that water may dissociate on $\mathrm{SiO}_{2}$ surfaces resulting in the formation of silanol $(\mathrm{Si}-\mathrm{OH})$ groups. These silanol groups can be detected in spectroscopic experiments via the $\mathrm{Si}-\mathrm{OH}$ stretching mode near $3750 \mathrm{~cm}^{-1}$ [20, 21, 22]. In particular it is believed that the silanol groups are a result of the interaction of water

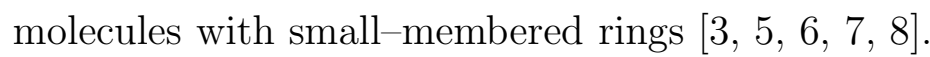

In this paper we investigate the reaction of a water molecule with a two-membered ring on an amorphous silica surface. To this end, we combine classical MD simulations using the so-called BKS potential [23] for silica and ab initio Car-Parrinello MD (CPMD) [24]. This combination of methods has recently also been used for investigating silicates in the bulk [25, 26] and of free silica surfaces [27]. The idea in our case is to generate configurations with a free surface by "BKS-MD" which are then the starting configurations for CPMD runs. The advantages of this methodology are two-fold: On the one hand, in the classical MD amorphous systems can be relaxed on a nanosecond time scale whereas typical time scales for the CPMD are only of the order of several picoseconds. On the other hand, the CPMD provides a much more realistic modeling of atomistic systems. This is of special importance in the case of interactions of water with an $\mathrm{SiO}_{2}$ surface since it is difficult to model these interactions realistically by classical potentials.

The rest of the paper is organized as follows: In the next section we describe how we have prepared a free silica surface by classical MD and give the details of the CPMD simulations. Then, the results for the water interaction with the two-membered ring are presented in Sec. 3. Finally, we summarize the results. 


\section{PREPARATION OF THE SURFACE AND DETAILS OF THE SIMULATIONS}

In order to study the reaction of a silica surface with a water molecule, we first have to prepare $\mathrm{SiO}_{2}$ configurations with a free surface. As we have already mentioned in the Introduction we use for this a combination of classical MD and CPMD.

As a model potential for the classical MD we use the so-called BKS potential [23] which is a simple pair potential of the following form:

$$
\phi(r)=\frac{q_{\alpha} q_{\beta} e^{2}}{r}+A_{\alpha \beta} \exp \left(-B_{\alpha \beta} r\right)-\frac{C_{\alpha \beta}}{r^{6}} \quad \alpha, \beta \in[\mathrm{Si}, \mathrm{O}],
$$

where $r$ is the distance between an atom of type $\alpha$ and an atom of type $\beta$. The effective charges are $q_{\mathrm{O}}=-1.2$ and $q_{\mathrm{Si}}=2.4$, and the parameters $A_{\alpha \beta}, B_{\alpha \beta}$, and $C_{\alpha \beta}$ can be found in the original publication. They were determined by using a combination of ab initio calculations and classical lattice dynamics simulations. The long-ranged Coulomb forces (and the potential) were evaluated by means of the Ewald summation technique. As an integrator for the simulation we used the velocity form of the Verlet algorithm [28] with a time step of 1.2 fs.

In order to investigate a system with free surfaces one could consider a film geometry, i.e. a system with periodic boundary conditions (PBC) in two directions and free boundaries in the third direction. Unfortunately, this is not a very good approach since the Ewald summation technique for the long ranged Coulomb interactions becomes inefficient in this case. This stems from the fact that the Fourier part of the Ewald sums can no longer be calculated by a single loop over the number of particles $N$ as in the case of $\mathrm{PBC}$ in all three directions, but one has to compute a double loop that scales with $N^{2}$ [29, 30, 31]. Therefore, we have adopted in this work a different strategy in that a system with PBC in all three directions was simulated containing an empty space $\Delta z$ in $z$-direction.

The preparation of the interface is done by the following steps: i) We start with a system at $T=3400 \mathrm{~K}$ with $\mathrm{PBC}$ in all three directions (box dimensions: $L_{x}=L_{y}=11.51 \AA$ and $L_{z}^{\prime}=23 \AA$ ). This system is fully equilibrated within 1 ns (see also below). As a result, configurations are obtained that indicate a realistic modelling of $\mathrm{SiO}_{2}$ with the empirical BKS potential, Eq. (11): A tetrahedral Si-O network is formed containing defects (e.g. fivefold coordinated silicon atoms) which are expected at a temperature as high as $T=3400 \mathrm{~K}$. However, no artificial bonds such as $\mathrm{Si}-\mathrm{Si}$ or $\mathrm{O}-\mathrm{O}$ bonds are found in the resulting network 
structures. ii) We cut the system perpendicular to the $z$-direction into two pieces. This is only done for oxygen-silicon bonds such that we get only free oxygen atoms at this interface. iii) These free oxygen atoms are now saturated by hydrogen atoms. The place of these hydrogen atoms is chosen such that each of the new oxygen-hydrogen bonds is in the same direction as the oxygen-silicon bonds which were cut and have a length of approximately $1 \AA$. The interaction between the hydrogen and the oxygen atoms as well as the silicon atoms are described only by a Coulombic term. The value of the effective charge of the hydrogen atoms is set to 0.6 which ensures that the system is still (charge) neutral. iv) We permanently freeze atoms which have a distance from the interface that is less than $4.5 \AA$, whereas atoms that have a larger distance can propagate subject to the force field, thus generating a mobile layer of $14.5 \AA$. v) We add in $z$-direction an empty space $\Delta z=6.0 \AA$. With this sandwich geometry we can use periodic boundary conditions in all three directions. We have made checked that the value of $\Delta z$ is sufficiently large to ensure that the structure of the free surface is not affected by the immobilized part of the system [32]. Finally, we have a system of 91 oxygen, 43 silicon, and 10 hydrogen atoms in a simulation box with $L_{x}=L_{y}=11.51 \AA$ and $L_{z} \approx 25 \AA$.

We have done 125 independent runs where we have equilibrated the system for 1 ns. For the present study we have selected from these runs one configuration that has exactly one two-membered ring on its surface. This configuration was then quenched to $T=300 \mathrm{~K}$ using a cooling rate of $2.8 \times 10^{12} \mathrm{~K} / \mathrm{s}$ where it was further relaxed for $0.25 \mathrm{~ns}$. Subsequently the final configuration of this run has been used as a starting point for the CPMD.

In the CPMD, we used conventional pseudopotentials for silicon and oxygen and the BLYP exchange functions [33, 34]. The electronic wave-functions were expanded in a plane wave basis set with an energy cutoff of $60 \mathrm{Ry}$ and the equations of motion were integrated with a time step of 0.085 fs for 0.2 ps. In the analysis of the CPMD run only those configurations were taken into account that were produced later than 5 fs after the start of the CPMD run in order to allow the system to equilibrate at least locally [25].

\section{RESULTS}

On intermediate length scales the surface structure of $\mathrm{SiO}_{2}$ can be well distinguished from

its bulk structure by the ring distribution [35]. A ring is defined as the shortest closed loop 
a)

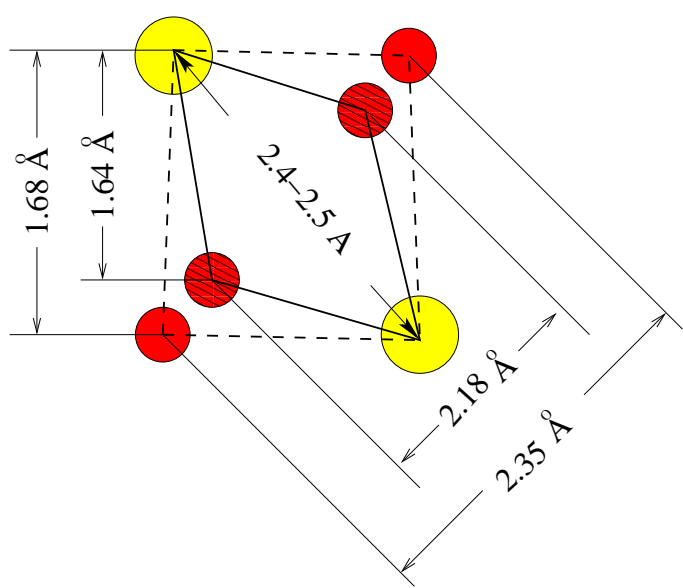

b)

$\mathrm{n}=3$

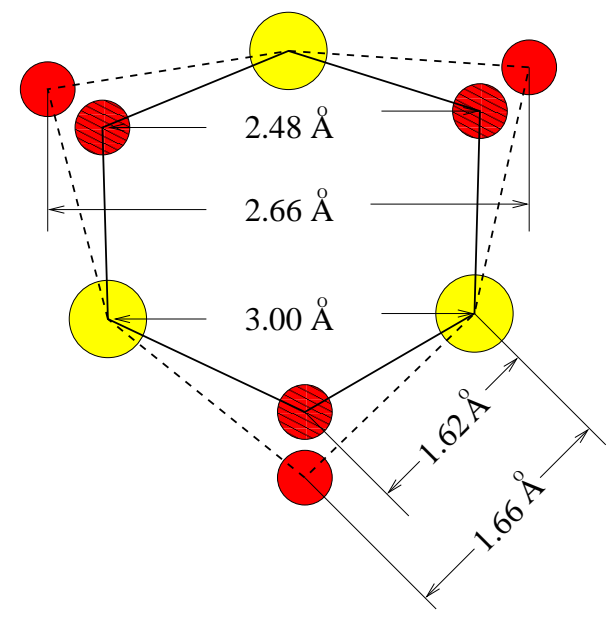

FIG. 1: Geometry of a) two-membered rings and b) three-membered rings as obtained from BKSMD (indicated by solid lines) and from CPMD (indicated by the dashed lines). Si atoms are shown as light circles and $\mathrm{O}$ atoms as small dark circles. For the sake of clarity of the figure, distances and angles are not to scale, and hence the difference between BKS-MD and CPMD in the figure is somewhat exaggerated in comparison with reality.

of $n$ consecutive $\mathrm{Si}-\mathrm{O}$ elements. The $\mathrm{SiO}_{2}$ surfaces that were obtained from the BKS-MD at $T=3400 \mathrm{~K}$ showed a relatively high concentration of $2-$ membered rings, i.e. edge-sharing tetrahedra, in contrast to the bulk in which, even at high temperatures, such structures are basically absent. The relaxation of the surfaces by CPMD reduced the number of $2-$ membered rings significantly at $T=3400 \mathrm{~K}$. At $T=300 \mathrm{~K}$, the time scale on which the system is relaxed by means of CPMD (0.2 ps) is not sufficient to cause a rearrangement of ring structures and thus the number of 2-membered rings does not change during the CPMD [27, 32]. However, both at $T=3400 \mathrm{~K}$ and at $T=300 \mathrm{~K}$, the CPMD yielded a slightly different geometry of 2-membered rings which is illustrated in Fig. [1. For BKS-MD as well as CPMD one finds a nearly planar geometry of two-membered rings. Whereas in CPMD a nearly quadratic shape with an $\mathrm{O}-\mathrm{Si}-\mathrm{O}$ angle around $90^{\circ}$ is seen, the BKS-MD leads to a trapezoid geometry with an $\mathrm{O}-\mathrm{Si}-\mathrm{O}$ angle around $80^{\circ}$. This is due to the fact that the distance between nearest $\mathrm{Si}-\mathrm{O}$ and $\mathrm{O}-\mathrm{O}$ neighbors is larger in CPMD while the distance between the silicon atoms in the 2 -fold ring is approximately around $2.5 \AA$ in both methods. A similar behavior can be seen for the 3-fold rings (see Fig. 1b) which exhibit 


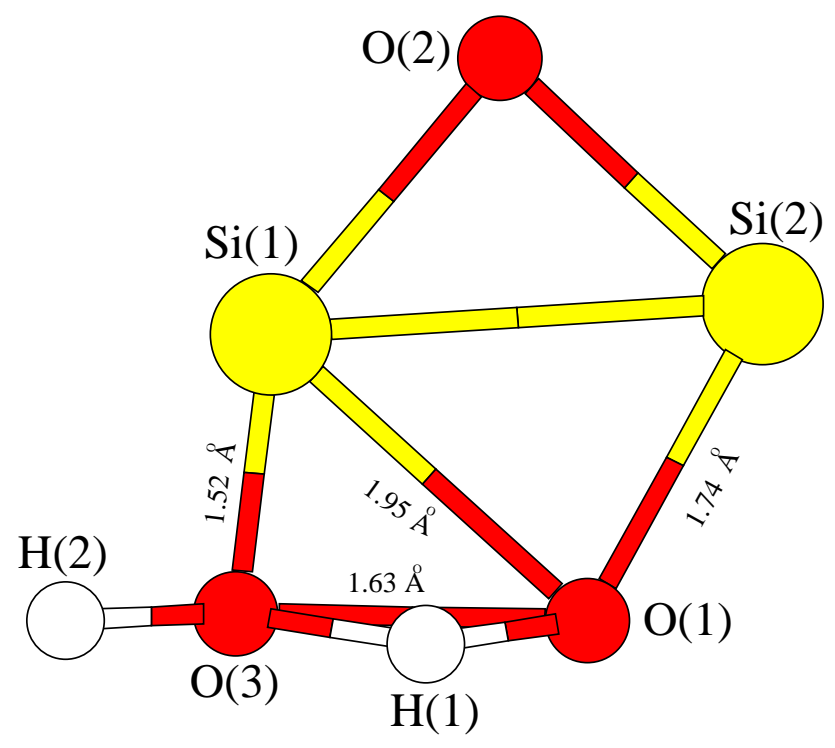

FIG. 2: Snapshot that illustrates the preparation of the water molecule on a two-fold ring. Note that $\mathrm{Si}(1), \mathrm{Si}(2), \mathrm{O}(1)$, and $\mathrm{O}(2)$ are part of the $\mathrm{Si}-\mathrm{O}$ network.

also a nearly planar geometry. Also, in this case BKS-MD and CPMD show a similar Si-Si distance (between nearest $\mathrm{Si}$ neighbors) around $3.0 \AA$ while the $\mathrm{Si}-\mathrm{O}$ distance is slightly larger in the CPMD. As a consequence, in the $\mathrm{BKS}-\mathrm{MD}$, the typical $\mathrm{O}-\mathrm{Si}-\mathrm{O}$ angle is $99^{\circ}$ whereas in the CPMD an $\mathrm{O}-\mathrm{Si}-\mathrm{O}$ of $107^{\circ}$ is found which is close to the angle of $109.47^{\circ}$ in an ideal tetrahedron.

Small-membered rings are of particular interest if one considers the reaction of a water molecule with an $\mathrm{SiO}_{2}$ surface. A possible reaction mechanism which was put forward by molecular orbital calculations [9, 10, 11] implies the disruption of a Si-O-Si structural element followed by the formation of two silanol groups $(\mathrm{Si}-\mathrm{O}-\mathrm{H})$. Thereby a $\mathrm{Si}-\mathrm{O}$ bond has to be broken. For this reason, 2- or 3-membered rings are of particular interest since these structures have a high local internal stress and hence their bonds can be broken more easily than the one in larger rings. Therefore, we consider in the following the reaction of $\mathrm{H}_{2} \mathrm{O}$ molecule with a $2-$ membered ring on a silica surface. To this end, we have selected a configuration from our BKS-CPMD simulations at $300 \mathrm{~K}$ that exhibit exactly one two-fold ring on its surface.

The initial position that we have chosen for the $\mathrm{H}_{2} \mathrm{O}$ molecule on the 2 -membered ring 


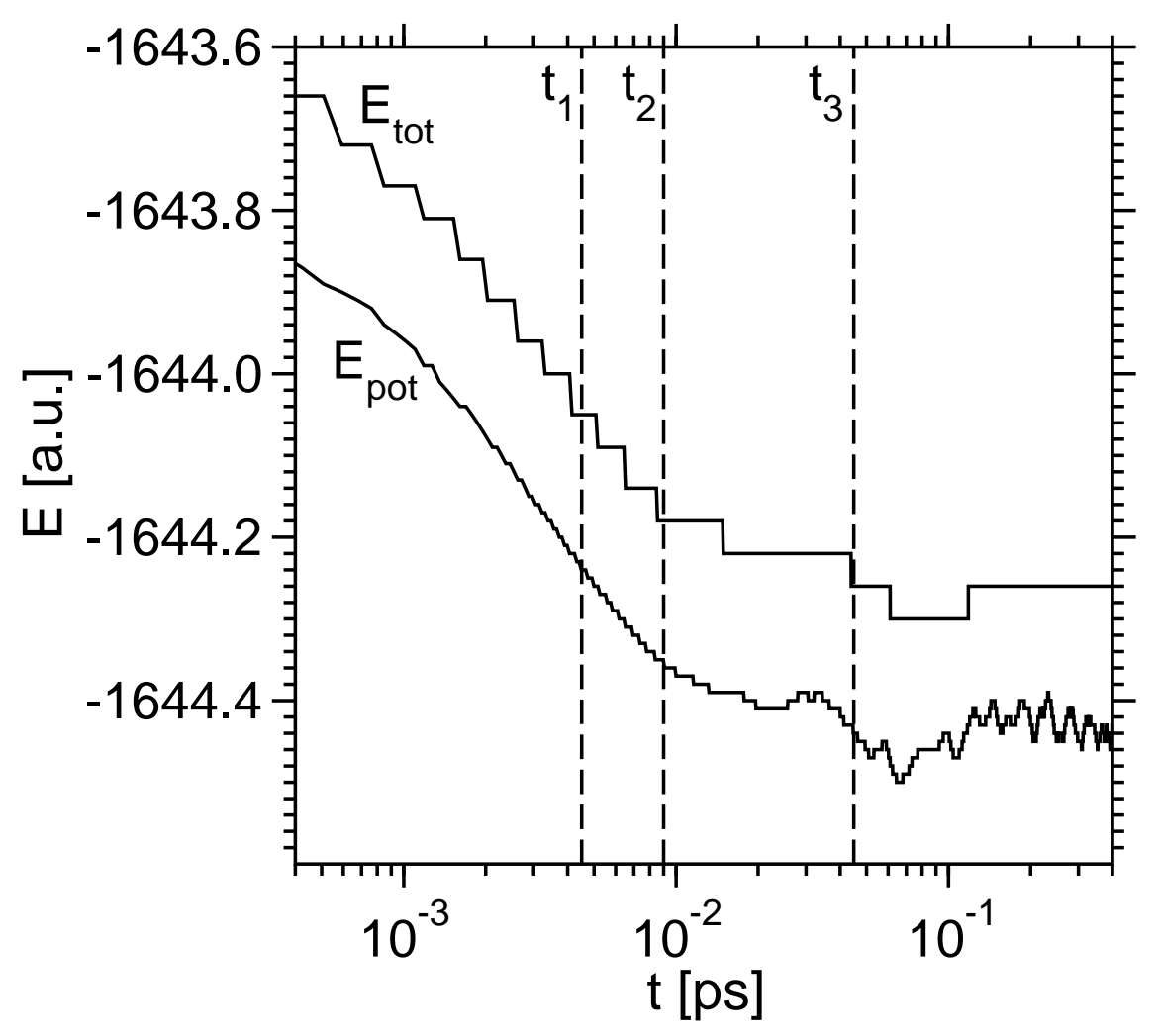

FIG. 3: Total energy $E_{\text {tot }}$ and potential energy $E_{\text {pot }}$ during the interaction of the silica surface with the $\mathrm{H}_{2} \mathrm{O}$ molecule (note the logarithmic time axis). The vertical dashed lines at $t_{1}=0.0045 \mathrm{ps}$, $t_{2}=0.009 \mathrm{ps}$, and $t_{3}=0.045 \mathrm{ps}$ mark the times at which the snapshots in Fig. 团 are shown.

is illustrated in Fig. 2] The oxygen atom of the water molecule $[\mathrm{O}(3)]$ was placed at a distance of $1.52 \AA$ from one of the $\mathrm{Si}$ atoms $[\mathrm{Si}(1)]$ which is slightly smaller than the $\mathrm{Si}-\mathrm{O}$ bond lengths in the ring of $1.64 \AA$. The two hydrogen atoms $H(1)$ and $H(2)$ were placed at a distance of $1 \AA$ from $\mathrm{O}(3)$ (and $\mathrm{H}(1)$ at a distance of $1 \AA$ from $\mathrm{O}(1)$ ) such that the $\mathrm{H}(1)-\mathrm{O}(3)-\mathrm{H}(2)$ angle corresponds to $109^{\circ}$. Further details can be extracted from Fig. 2. We shall see in the following that the small $\mathrm{O}(1)-\mathrm{O}(3)$ distance $(1.63 \AA$ compared to $2.2 \AA$ in bulk silica) drives the breaking of a $\mathrm{Si}-\mathrm{O}$ bond and the formation of two silanol groups.

The initial condition for $\mathrm{H}_{2} \mathrm{O}$ on the two-membered rings is of course artificial and leads to enormous forces between the atoms. In order to avoid an explosion of the system we have introduced a thermostat. If the temperature becomes larger than $380 \mathrm{~K}$ or lower than $220 \mathrm{~K}$, the velocities of the particles are rescaled such that the apparent temperature of the system is $300 \mathrm{~K}$. Figure 3 shows the potential and the total energy during the reaction whereby the time $t=0$ corresponds to the initial configuration. Obviously, the potential 
a)

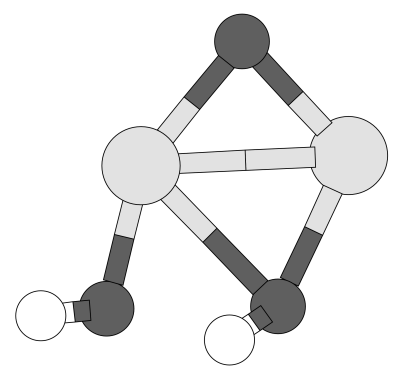

b)

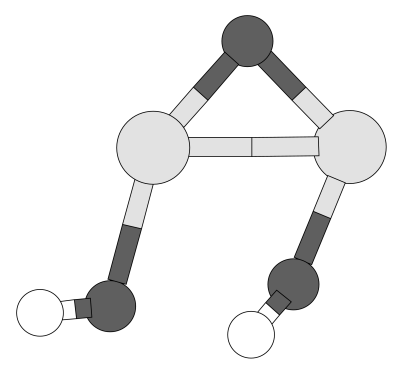

c)

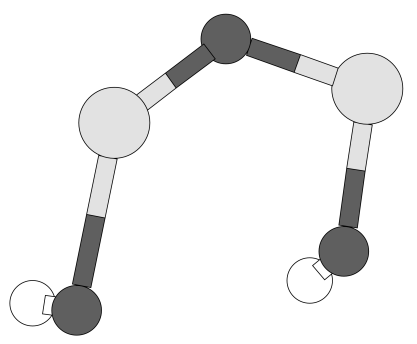

FIG. 4: Snapshots of the reaction of the $\mathrm{H}_{2} \mathrm{O}$ molecule with the 2 -fold ring at a) $t=t_{1}$, b) $t=t_{2}$, and c) $t=t_{3}\left(t_{1}, t_{2}\right.$, and $t_{3}$ as indicated in Fig. (3).

energy decreases rapidly such that after about $0.1 \mathrm{ps}$ a constant is reached. Snapshots of the reaction at $t_{1}=0.0045 \mathrm{ps}, t_{2}=0.009 \mathrm{ps}$, and $t_{3}=0.045 \mathrm{ps}$ are shown in Fig. 团 (note that $t_{1}, t_{2}$, and $t_{3}$ are also marked as vertical dashed lines in Fig. (3). Indeed, at time $t_{3}$ the end configuration with two silanol groups is formed. The first step that leads to the silanol groups is the breaking of one of the $\mathrm{H}-\mathrm{O}$ bonds and of the enforced $\mathrm{O}(3)-\mathrm{O}(1)$ bond. This has happened at $t=t_{1}$ whereby the potential energy is already quite close to the equilibrium value (see Fig. 31). In a second step then a $\mathrm{Si}-\mathrm{O}$ bond is broken (see snapshot at $t=t_{2}$ ) followed by an increased separation of the two silicon atoms.

Now we want to study in more detail the formation of two silanol groups as a result of the reaction of the water molecule with a two-fold ring. This reaction can be written as follows:
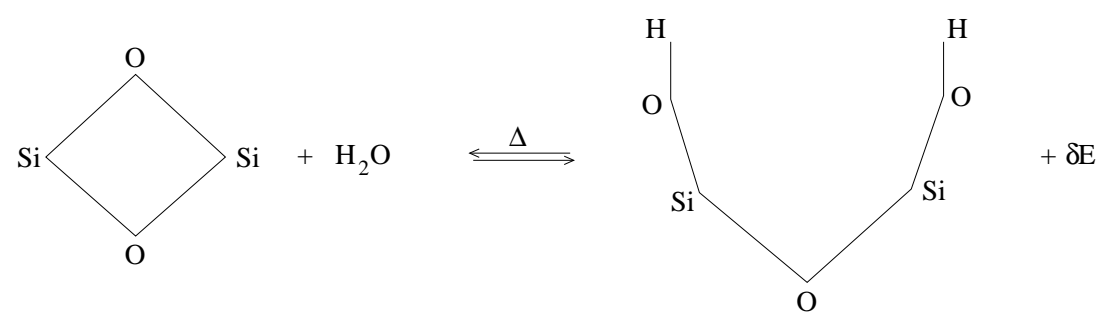

In the following we will estimate the activation energy $\Delta$ and also $\delta E$. A positive or negative sign of $\delta E$ indicates whether the reaction is exothermic or endothermic, respectively. We use again the starting configuration shown in Fig. 2. But now we completely freeze in the $\mathrm{SiO}_{2}$ system and we move only the water molecule at a temperature of $30 \mathrm{~K}$. As the inset of Fig. 5 shows, the potential energy is monotonously decaying while the water molecule relaxes on 

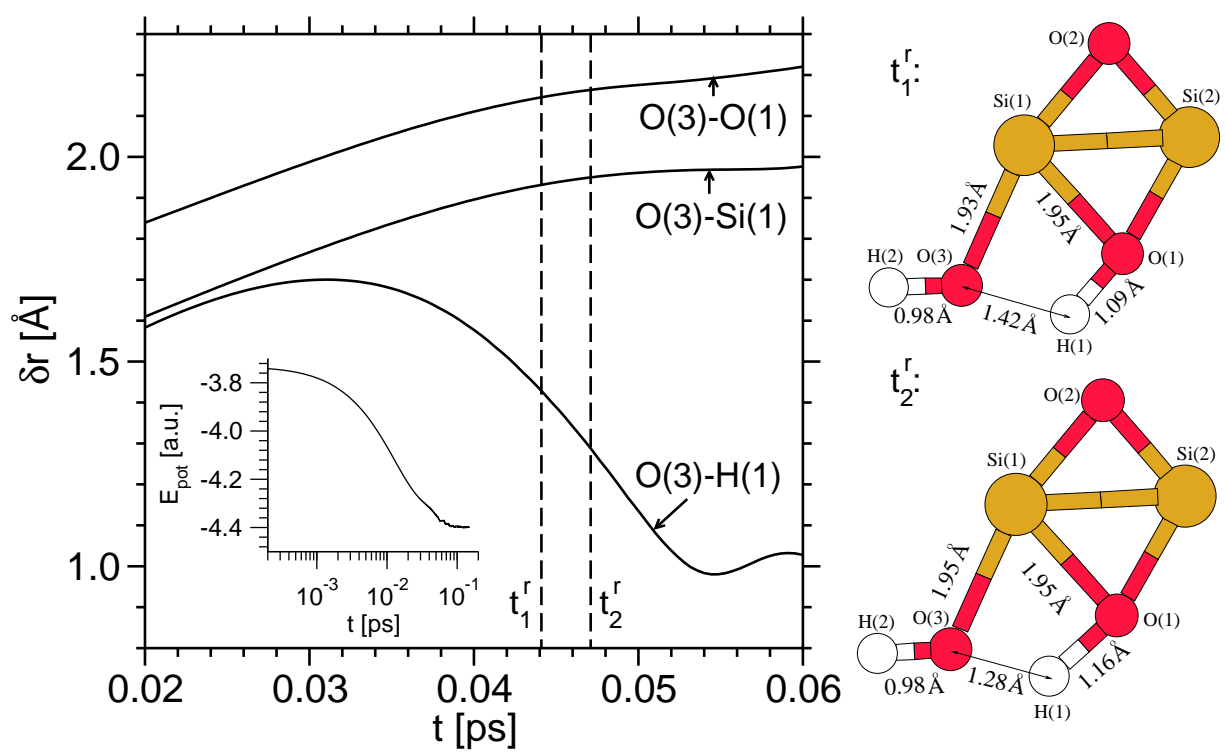

FIG. 5: Change in the distance $\delta r$ between the indicated atoms (that are defined in the two snapshots at times $t_{1}^{\mathrm{r}}=0.044 \mathrm{ps}$ and $t_{2}^{\mathrm{r}}=0.047 \mathrm{ps}$ ) during the simulation at $T=30 \mathrm{~K}$ where only the $\mathrm{H}_{2} \mathrm{O}$ molecule was moved whereas the other atoms were fixed. The inset shows the potential energy for the latter simulation (note that $E_{\text {pot }}$ is shifted by 1640 a.u.).

the surface. After about $0.1 \mathrm{ps}$ a constant value of $E_{\text {pot }}$ is reached where the water molecule has evaporated from the surface. Also shown in Fig. 5 are the distances $\delta r$ of the water atoms from the initial position as a function of time. We see that, slightly before the $\mathrm{H}_{2} \mathrm{O}$ evaporates, the $\mathrm{O}(3)-\mathrm{O}(1)$ distance and the $\mathrm{O}(3)-\mathrm{Si}(1)$ distance are increasing while the $\mathrm{O}(3)-\mathrm{H}(1)$ distance is decreasing to the ideal $\mathrm{O}-\mathrm{H}$ distance in a water molecule around $1 \AA$. Of importance for the following, are the configurations at the times $t_{1}^{\mathrm{r}}=0.044 \mathrm{ps}$

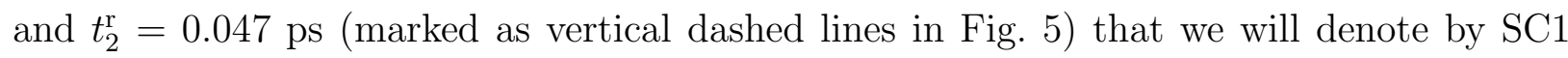
and $\mathrm{SC} 2$, respectively. Using $\mathrm{SC} 1$ as a starting configuration for a CPMD run, where the whole system is again simulated at $T=300 \mathrm{~K}$, leads to the formation of two silanol groups, whereas the use of SC2 yields the evaporation of the water molecule from the surface. As

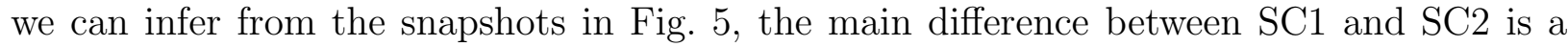
slightly different $\mathrm{O}(3)-\mathrm{H}(1)$ distance.

In Fig. [6 we plot the potential energy for the two CPMD runs starting from the configurations $\mathrm{SC} 1$ and $\mathrm{SC} 2$. Since both runs yield initially about the same value for $E_{\mathrm{pot}}$ we can extract both $\Delta$ and $\delta E$ from the plot. First, from the difference of the saturated values for $t>0.1 \mathrm{ps}$ (marked as horizontal lines in Fig. [6) we obtain $\delta E=E\left(\mathrm{SiO}_{2}+\mathrm{H}_{2} \mathrm{O}\right)-E(2 \mathrm{SiOH}) \approx$ 


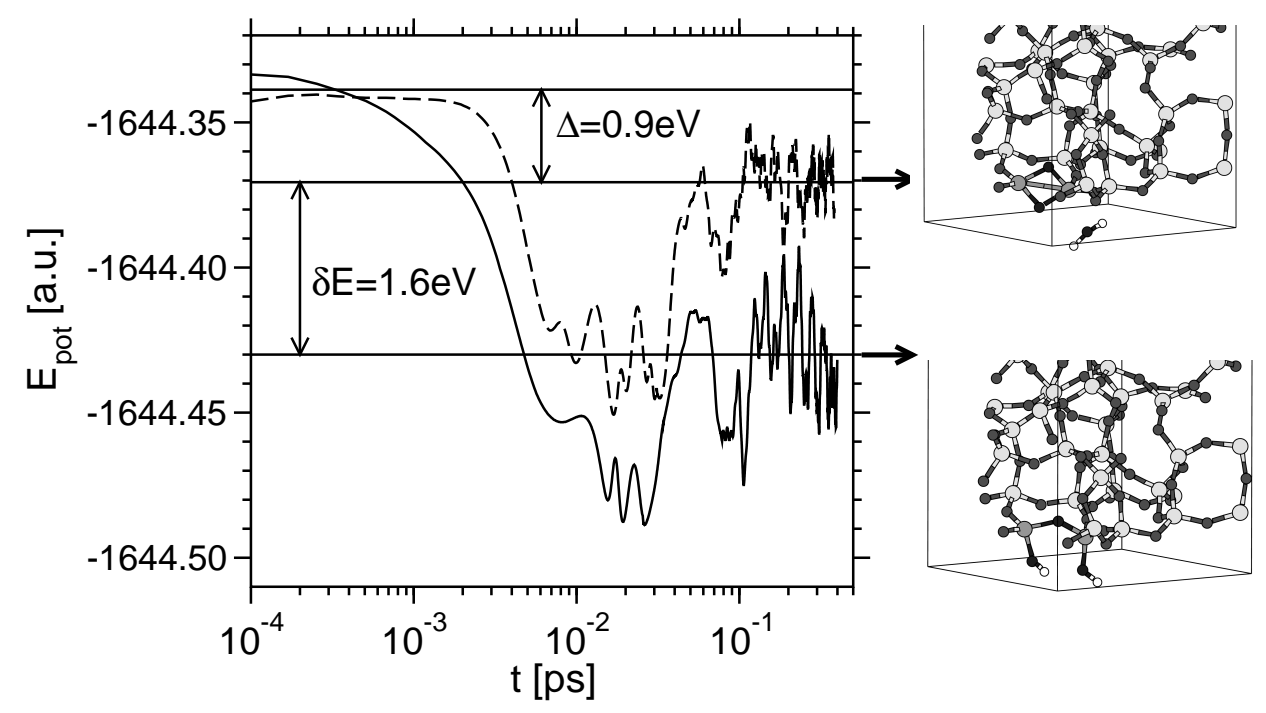

FIG. 6: Potential energy as a function of time for the simulations at $T=300 \mathrm{~K}$ using the start configuration SC2 (dashed line) and SC1 (solid line). The snapshots correspond to the end configurations of the two runs as indicated. In both figures the two hydrogen atoms are shown as small white spheres at the bottom of the simulation box.

$1.6 \mathrm{eV}$ which indicates that the reaction is exothermic. The activation energy $\Delta$ can be estimated from the difference of the initial and the final value of the SC2 run where the water molecule evaporates from the surface. For the latter process an $\mathrm{HO}-\mathrm{H}$ bond and a $\mathrm{Si}-\mathrm{O}$ bond have to be broken and thus the binding energy of both bonds contribute to $\Delta$. From Fig. [6 the value $\Delta \approx 0.9 \mathrm{eV}$ can be extracted. Whereas the distances of the $\mathrm{O}-\mathrm{O}$ and $\mathrm{Si}-\mathrm{O}$ neighbors in the two-membered ring are significantly smaller than those found in the bulk (see above), the same bonds are very close to the bulk values in the final structure with the two silanol rings. Moreover, the $\mathrm{O}-\mathrm{Si}-\mathrm{O}$ angle in the silanol groups has a value around $108^{\circ}$, i.e. close to the value found in an ideal $\mathrm{SiO}_{4}$ tetrahedron. These findings may explain the observation of an exothermic reaction.

In a recent publication, Masini and Bernasconi [12] also studied the adsorption reaction of a water molecule with a two-membered ring on an amorphous silica surface by CPMD. They find $\delta E \approx 1.7 \mathrm{eV}$, i.e. a value which is very similar to the one found in this work. However, they did a constrained MD simulation to estimate the activation energy. As a reaction coordinate they used either the distance between a silicon atom in the ring and the oxygen atom of the water molecule (called path A in Ref. [12]) or the distance between an 
oxygen atom of the ring and a hydrogen atom of the water molecule (called path B). As a result $\Delta=1.1 \mathrm{eV}$ is obtained for path $\mathrm{A}$ and $\Delta=0.32 \mathrm{eV}$ for path $\mathrm{B}$. In this work, we find an activation energy which is closer to path $\mathrm{A}$ in Ref. [12].

\section{SUMMARY}

Using a combination of classical MD and CPMD we have investigated the reaction of water with a free amorphous silica surface. We have seen that the reaction of a water molecule with a two-membered ring, leading to the formation of two silanol groups on the $\mathrm{SiO}_{2}$ surface, is an exothermic reaction with an activation energy around $0.9 \mathrm{eV}$. That the reaction is exothermic is in agreement with other recent numerical studies, and may be

explained by the change of bond lengths and angles towards values found in bulk $\mathrm{SiO}_{2}$ while the silanol groups form.

\section{Acknowledgements}

We are grateful to Michele Parrinello and Gloria Tabacchi for the introduction of one of us (C. M.) into CPMD. Without their support the present work would not have been possible. We acknowledge financial support by the SCHOTT Glaswerke Fond, the DFG under SFB 262, the BMBF under grant No. 03N6015, and the European Community's Human Potential Program under contract HPRN-CT-2002-00307, DYGLAGEMEM. J. H. acknowledges financial support from the DFG under grants HO 2231/2-1/2. We thank the NIC Jülich for a generous grant of computing time.

[1] A. P. Legrand, The Surface Properties of Silica, Wiley, New York, 1998.

[2] R. K. Iler, The Chemistry of Silica, Wiley, New York, 1979.

[3] B. A. Morrow, I. A. Cody, J. Phys. Chem., 1976, 80, 1995.

[4] T. A. Michalske, B. C. Bunker, J. Appl. Phys., 1984, 56, 2686.

[5] B. C. Bunker, D. M. Haaland, K. J. Ward, T. A. Michalske, W. L. Smith, J. S. Binkley, C. F. Melius, C. A. Balfe, Surf. Sci., 1989, 210, 406.

[6] L. H. Dubois, B. R. Zegarski, J. Am. Chem. Soc., 1993, 115, 1190. 
[7] L. H. Dubois, B. R. Zegarski, J. Phys. Chem., 1993, 97, 1665.

[8] A. Grabbe, T. A. Michalske, W. L. Smith, J. Phys. Chem., 1995, 99, 4648.

[9] M. O'Keeffe, G. V. Gibbs, J. Chem. Phys., 1984, 81, 876.

[10] T. Uchino, Y. Tokuda, T. Yoko, Phys. Rev. B, 1998, 58, 5322.

[11] I. S. Chuang, G. E. Maciel, J. Am. Chem. Soc., 1996, 118, 401.

[12] P. Masini, M. Bernasconi, J. Phys.: Condens. Matter, 2002, 14, 4133.

[13] M. H. Du, L. L. Wang, A. Kolchin, H. P. Cheng, Eur. Phys. J. D, 2003, 24, 323.

[14] M. H. Du, A. Kolchin, H. P. Cheng, J. Chem. Phys., 2003, 119, 6418.

[15] M. H. Du, A. Kolchin, H. P. Cheng, J. Chem. Phys., 2004, 120, 1044.

[16] E. B. Webb, S. H. Garofalini, J. Non-Cryst. Sol., 1998, 226, 47.

[17] V. A. Bakaev, W. A. Steele, J. Chem. Phys., 1999, 111, 9803.

[18] M. Wilson, T. R. Walsh, J. Chem. Phys., 2000, 113, 9180.

[19] T. R. Walsh, M. Wilson, A. P. Sutton, J. Chem. Phys., 2000, 113, 9191.

[20] M. L. Hair, Infrared Spectroscopy in Surface Chemistry, Dekker, New York, 1967.

[21] P. R. Ryason, B. G. Russell, J. Phys. Chem., 1975, 79, 1276.

[22] B. A. Morrow, A. J. McFarlan, J. Non-Cryst. Sol., 1990, 120, 61.

[23] B. W. H. van Beest, G. J. Kramer, R. A. van Santen, Phys. Rev. Lett. 1990, 64, 1955.

[24] R. Car, M. Parrinello, Phys. Rev. Lett. 1985, 55, 2471.

[25] M. Benoit, S. Ispas, P. Jund, R. Jullien, Eur. Phys. J. B 2000, 13, 631.

[26] S. Ispas, M. Benoit, P. Jund, R. Jullien, Phys. Rev. B, 2001, 64, 214206.

[27] C. Mischler, W. Kob, K. Binder, Comp. Phys. Comm., 2002, 147, 222.

[28] M. P. Allen, D. Tildesley, Computer Simulation of Liquids, Clarendon Press, Oxford, 1987.

[29] D. E. Parry, Surf. Sci., 1975, 49, 433.

[30] D. E. Parry, Surf. Sci., 1976, 54, 195.

[31] S. W. de Leeuw and J. W. Perram, Physica A, 1982, 113A, 546.

[32] C. Mischler, Molekulardynamik-Simulation zur Struktur von SiO ${ }_{2}$-Oberflächen mit adsorbiertem Wasser, Ph.D. Thesis, Mainz, 2002.

[33] N. Trouiller, J. L. Martins, Phys. Rev. B, 1991, 43, 1993.

[34] C. Lee, W. Yang, R. G. Parr, Phys. Rev. B, 1988, 37, 785.

[35] A. Roder, W. Kob, K. Binder, J. Chem. Phys., 2001, 114, 7602. 